This is a self-archived version of an original article. This version may differ from the original in pagination and typographic details.

Author(s): Pesonen, Heidi; Savic, Andrej M.; Kujala, Urho; Tarkka, Ina

Title: Long-term physical activity modifies automatic visual processing

Year: 2019

Version: Accepted version (Final draft)

Copyright: @ International Society of Sport Psychology 2019

Rights: In Copyright

Rights url: http://rightsstatements.org/page/InC/1.0/?language=en

Please cite the original version:

Pesonen, H., Savic, A. M., Kujala, U., \& Tarkka, I. (2019). Long-term physical activity modifies automatic visual processing. International Journal of Sport and Exercise Psychology, 17(3), 275284. https://doi.org/10.1080/1612197X.2017.1321031 


\section{Long-term physical activity modifies automatic visual processing}

Heidi Pesonen $^{\mathrm{a}}$, Andrej M. Savićc ${ }^{\mathrm{c}}$, Urho M. Kujala ${ }^{\mathrm{a}}$ and Ina M. Tarkka ${ }^{\mathrm{a}}$

${ }^{\text {a} D e p a r t m e n t ~ o f ~ H e a l t h ~ S c i e n c e s, ~ U n i v e r s i t y ~ o f ~ J y v a ̈ s k y l a ̈, ~ J y v a ̈ s k y l a ̈, ~ F i n l a n d ; ~ ' ~}{ }^{\text {SSignals and }}$ Systems Department, University of Belgrade, Belgrade, Serbia 'Tecnalia Serbia Ltd., Belgrade, Serbia

\section{Abstract}

Electrophysiologically registered visual mismatch negativity (vMMN) is known to represent automatic visual processing in human visual cortex. Since physical activity (PA) is generally beneficial to cerebrovascular function (Nishijima, Torres-Aleman, \& Soya, 2016) we wanted to find out if automatic visual processing is affected by PA. We investigated the connection between long-term leisure-time PA and precognitive visual processing in 32 healthy young males. Participants were divided into active $(n=16)$ and inactive $(n=16)$ group according to their leisure-time PA records from the past three years. VMMN was recorded with electroencephalogram using passive oddball paradigm with visual bars. Standard (90\%) and deviant $(10 \%)$ stimuli in different orientations were presented randomly while participant's attention was directed to an audio play. No visual task was involved. VMMN difference waveforms were generated and peak latencies and signal integrals were determined in poststimulus window of 100-300 ms. VMMN latencies were shorter in active participants compared to inactive ones in the occipital cortex. A trend toward larger integral values in occipital area in active participants was observed, albeit non-significant. Physically active participants showed faster automatic processing of deviant stimuli compared to inactive ones in the occipital area. This may imply enhanced early non-attended visual processing in those individuals who are habitually physically active. 
Key words: visual mismatch negativity, physical exercise, distractibility, healthy young males 


\section{Introduction}

When performed regularly, moderate to vigorous intensity physical activity (PA) is widely known to have major health benefits including reduced levels of total and visceral fat, reduced risk of metabolic syndrome, type 2 diabetes and an association with reduced incidence of fatal coronary heart disease (WHO, 2010). Recently increasing evidence has suggested that PA already in young age has an effect on cognitive function (Haapala et al., 2016; Hillman, Erickson, \& Kramer, 2008; Themanson \& Hillman, 2006; van Praag, Fleshner, Schwartz, \& Mattson, 2014). For instance, Themanson \& Hillman (2006) showed that cardiorespiratory fitness was associated with better cognitive performance and action monitoring (Themanson \& Hillman, 2006). Aerobic fitness and long term physical activity in general have been associated with better cognitive functioning. However, the majority of those studies are conducted for the aging population (Andel et al., 2008; Erickson et al., 2009; Voelcker-Rehage \& Niemann, 2013). Studies investigating associations of PA and brain function in young adults mostly perform comparisons with older adults (Andel et al., 2008; Erickson et al., 2009; Voelcker-Rehage \& Niemann, 2013). This is understandable, because cognitive decline in ageing is a major factor in quality of life and young adulthood is considered to be a time of good cognitive health (Hillman et al., 2008; Voelcker-Rehage \& Niemann, 2013). However, inactive lifestyle is increasingly common in all ages and therefore the effects of PA on brain functions should be studied also in young adults in their peak cognitive health. 
In recent years, research has been able to identify changes in neurotransmitters, neurotrophins, fine neuronal morphology, angiogenesis and hippocampal neurogenesis as a result of regular exercise (van Praag et al., 2014). PA has also been found to be an effective method in enhancing cognitive functions, such as memory, attention, processing speed and executive functions (Rottensteiner et al., 2015; Smith et al., 2010). Specific cortical functions underlying good cognitive performance associated with PA, have gained interest as well, but those are not yet fully understood (Donnelly et al., 2016; Hillman et al., 2008; VoelckerRehage \& Niemann, 2013). Particularly highly specialized precognitive sensory functions are seldom associated with measures of active lifestyle. Visual processing is recognized as an important sensory function, which involves large part of cerebral cortex. The scalp-recorded event-related potential registering visual precognitive change detection, called the visual mismatch negativity (vMMN), is well established and is thought to represent human precognitive non-attended visual processing in the visual cortical and prefrontal areas (Czigler, Weisz, \& Winkler, 2007; Kimura, 2012; Kremlacek et al., 2016). Such processing is yet to be connected to PA in previous research and thus we believe that vMMN could serve as a sensitive marker of modifiability of automatic visual processing.

Mismatch negativity (MMN) was first discovered in the auditory system (Naatanen, Gaillard, \& Mantysalo, 1978) and is most commonly investigated by a passive oddball paradigm. This experimental paradigm has been utilized in visual modality by a variety of visual stimuli (Kremlacek et al., 2016). These have included color, location, luminance, orientation, spatial frequency, duration of the visual stimulus, motion direction, object formation or deformation and even emotional expressions (Czigler et al., 2007; Kimura, 2012; Kuldkepp, Kreegipuu, Raidvee, Naatanen, \& Allik, 2013; Pazo-Alvarez, Cadaveira, \& Amenedo, 2003). An essential part of eliciting a $\mathrm{MMN}$ is the independence of attention and it is clearly harder to 
achieve an attention-free situation when dealing with visual modality compared to auditory. Usually this is achieved by an auditory stimulus and task during the visual presentation (Stefanics, Kremlacek, \& Czigler, 2014). VMMN is visualized and measured as a difference waveform, calculated by subtracting the waveform elicited by standard stimuli from the waveform elicited by deviant stimuli. It is usually observed in occipito-temporal areas at 100400 ms range after stimulus (Kimura \& Takeda, 2015; Pazo-Alvarez et al., 2003).

VMMN was first explained by a memory trace in the brain created by the standard stimuli, and the mismatch between its representation and the representation of the deviant stimulus. This theory is considered to follow largely the MMN theory in the auditory modality (Czigler et al., 2007). Recently this theory has been replaced by a theory suggesting that the standard stimuli create a prediction of events and the occurring incongruent event breaks this prediction, non-consciously (Kimura \& Takeda, 2015; Stefanics et al., 2014). Ability to detect an event that does not match prediction is probably critical to our survival. For example, fast changing events in modern traffic necessitate high-speed automatic visual processing. In the visual domain vMMN detects this type of processing, and it has not yet been connected with PA. Furthermore, visual processing and vMMN have been connected with better cognitive functions (Stefanics et al., 2014), and better cognitive functions have been related to regular PA (Hillman et al., 2008; Smith et al., 2010; van Praag et al., 2014). The purpose of this study was to investigate the relationship between long term leisure-time PA and non-attended precognitive visual processing (vMMN) in young healthy males. We hypothesized that PA may enhance vMMN. This enhancement could possibly be related to the fact that PA is shown to generally enhance synaptic plasticity, neurogenesis and cerebral vascular function (Nishijima et al., 2016; van Praag et al., 2014). 


\section{Methods}

\section{Participants}

Study participants were 32 young healthy males (aged 32-37 y), who were part of the FITFATTWIN study population. Initially male twins were recruited from a population-based, longitudinal Finntwin16 cohort study based on their answers about leisure-time PA (Kaprio, Pulkkinen, \& Rose, 2002). The discordance in leisure-time activity was determined based on the frequency of their leisure-time PA with two different inclusion criteria: (1) the more active co-twin was physically active $\geq 2$ times per week, and the less active co-twin of the same pair $\leq 2$ times per month; (2) the more active co-twin participated $\geq 2$ times per week in PA at an intensity equivalent to easy or brisk running, and the PA of the less active co-twin of the same pair needed to be less intense and less frequent or shorter duration. The second inclusion criterion was used if the first criterion was not met. Ethical approval was obtained from the local review board for human research and all participants gave a written informed consent. Exclusion criteria included specific chronic diseases or a heavy use of tobacco, alcohol or medication. After screening process, potential participants were interviewed by telephone with questions about their current health and physical activity habits during the past three years. At this point exclusion criteria included either declining to take part in the study, having specific acute disease affecting ability to be physically active, major changes in PA or failure to take part in the telephone interview (Rottensteiner et al., 2015). As the mean age of the participants was about 35 years and we wanted to identify pairs who were long-term discordant for PA and investigate the effects of PA, we studied only men because before this age pregnancies have a major influence on physical activity fluctuations and pregnancies as well as fluctuations related to menstrual cycle may also have an influence on biological parameters targeted in the present study. 
After the screening process 16 pairs went through a comprehensive clinical study measurements and detailed PA interviews. Nine pairs were classified as significantly discordant for leisure-time PA based on interview. Further seven pairs, concordant within each pair for PA, were included in the study to gain a larger population. Participants were divided into two groups according to their leisure-time PA level individually, regardless of their twin status. Those participants that had their daily average MET-index (Metabolic Equivalent of Task -hours/day, indicating volume of leisure-time PA) $\geq 2$ for the past three years (described below) were placed in the active group $(n=16)$, and those participants that had $<2$ daily MET-index for the past three years were placed in the inactive group $(n=16)$.

\section{Experiments}

The amount of each participant's leisure-time PA was measured as described previously (Rottensteiner et al., 2015) with structured PA interviews, which included work journey activity. With a shorter retrospective PA interview leisure-time PA volume at one-year intervals over the past 6 years was assessed. The resulted volume of leisure-time PA was quantified as a leisure-time MET index, and calculated as frequency (per month) $\times$ duration (min) xintensity (MET) and work journey activity as frequency (five times per week) $\times$ duration ( $\mathrm{min}) \times$ intensity of 4 METs. The results were presented as sum score of METhours per day. The average leisure-time PA for the past three years was expressed as 3-year mean leisure time MET index. This was used as the group division criterion.

Clinical assessment included aerobic fitness test with braked bicycle ergometer and gas exchange analysis (Spiroergometer, Sensormedics, Yorba Linda, CA, USA). During the test the workload started at $25 \mathrm{~W}$ and was increased every two minutes by $25 \mathrm{~W}$ until exhaustion, or until the maximal exercise capacity was reached. Maximal exercise capacity was 
determined as either exertion of $19-20 / 20$ on Borg scale, or gas exchange ratio over 1.1. Maximal oxygen uptake $\left(\mathrm{VO}_{2} \max \right)$ was determined as the mean value of the two highest consecutive $\mathrm{VO}_{2}$ values recorded during periods of 30 s. Height and weight were measured. Waist circumference was measured. Whole body composition was measured with dualenergy x-ray absorptiometry (DEXA Prodigy; GE Lunar Corp., Madison, WI). The participants were instructed not to perform vigorous physical activity over the preceding two days before the measurements. This instruction was given to avoid short term adaptations to PA.

To obtain vMMN EEG recording was performed while participants listened to an audio play. They were instructed to fix their gaze at the cross in the middle of the screen placed at distance of $1.2 \mathrm{~m}$ in front of them at their eye level where stimuli were presented. They were asked not to pay attention to the visual stimuli but to attend to the audio play. The visual stimuli consisted of black bars each presented $100 \mathrm{~ms}$ on the screen in a light grey background. The bars of the standard stimuli were tilted $18^{\circ}$ to the right and the deviant stimuli $18^{\circ}$ to the left (Astikainen, Lillstrang, \& Ruusuvirta, 2008). One thousand stimuli were delivered, of which $10 \%$ were deviant stimuli. Stimuli were presented in a random order but with the restriction that at least two standard stimuli were always presented between deviant stimuli. Interstimulus interval was $1100 \mathrm{~ms}$.

EEG was recorded with Hydrogel Geodesics Sensor Net with 128-channels (Electrical Geodesics Inc., Eugene, OR, USA) using Cz reference. The sampling rate was $1000 \mathrm{~Hz}$ using $0.1 \mathrm{~Hz}-400 \mathrm{~Hz}$ bandpass. EEG was baseline corrected, filtered and segmented to $500 \mathrm{~ms}$ epochs (100 ms before the stimulus onset used for baseline), all noise-free deviants were averaged to form the deviant ERP waveform using custom routines written in Matlab 
(Mathworks, Natick, MA, USA). The number of accepted deviant epochs for averaging ranged from 48 to 99 among participants. Data from all participants were included in the analysis. For each participant, standard ERP waveform was created by averaging a subset of noise-free waveforms elicited by standard stimuli, randomly selected to match the number of epochs elicited by deviant stimuli. The difference ERP waveform of each channel was calculated by subtracting participant's waveforms elicited by standard stimuli from waveforms elicited by deviant stimuli.

\section{VMMN analysis}

First grand averaged standard, deviant and difference waveforms were created for each group and all-channel ERPs with topographic maps were plotted for visual inspection (6 timepoints of waveforms elicited by deviant stimuli are illustrated within the analyzed window, Fig.1.). In order to avoid multiple comparison problems, specific channels were selected based on topographic maps and previous knowledge of vMMN sources (Kimura, 2012; Kremlacek et al., 2016). As we have 128 channels, several channels overlay known source regions and thus we selected best representative channels 20 and 118 from the frontal region (approx. F3 and F4 electrode placements in International 10-20 System), and channels 71, 72 and 76 from the occipital region (approx. O1, Pz and O2). The difference waveforms of these channels were analyzed. First, peak latencies were detected within the time window of 100-300 ms. Waveforms were rectified and integral values within the window were statistically analyzed. The data processing was performed with Matlab (MathWorks, Natick, MA, USA). The statistical analysis was made with IBM SPSS statistics 20 (IBM Corporation, New York, NY, USA) using Shapiro-Wilk test for normality and Mann-Whitney U-test for group comparisons. Tables also indicate the tests used. 


\section{Results}

The groups indeed differed in their PA levels. In the active group $(n=16)$ daily average METindex was 7.8 \pm 5.24 and in the inactive group $(\mathrm{n}=16)$ it was $0.95 \pm 0.54 \quad(\mathrm{p}<.001)$. Anthropometrically groups were similar but active group had lower body fat $\%$ and waist circumference and higher $\mathrm{VO}_{2 \max }$ value in concordance with PA level (Table 1.).

(Table 1 and Fig. 1. about here)

Waveform plots and topographic maps suggested differences in visual processing in vMMN between groups. Topographic maps of the waveforms elicited by deviant stimuli revealed somewhat comparable configurations during early visual evoked potential (Fig. 1.). However, late component, identified with the peak latency of vMMN between 207- $255 \mathrm{~ms}$ according to previous literature (Astikainen et al., 2008; Kimura \& Takeda, 2015; Stefanics et al., 2014), clearly differed. For further analysis the standard, deviant and difference waveforms of the selected channels $(20,118,71,72$ and 76$)$ were taken. These channels in active and inactive groups are illustrated in Fig 2. VMMN was identified in difference waveforms as a negative component with peak latency between 207 and $248 \mathrm{~ms}$ in all participants. According to earlier literature "genuine vMMN" appears approximately in this time window. The difference waveform early component is not deviant-related negativity but direct visual evoked potential (Astikainen et al., 2008; Kimura, 2012; Stefanics et al., 2014). VMMN latency was calculated from the late component peak negativity of the difference waveform. The latencies were significantly shorter in the active group in the occipital cortex compared to the inactive group (channels: $71, \mathrm{p}=.001,72, \mathrm{p}=.023$ and $76, \mathrm{p}=.026$ ). No differences were seen between groups in frontal channels 20 and 118 (Table 2).

(Table 2 and Fig. 2. about here) 
The total vMMN activation was measured in waveform integrals in the rectified difference waveforms in the time window of 100-300 ms. There were no differences in integrals in frontal channels 20 and 118. The active group suggested a trend, albeit non-significant, for larger integral compared to the inactive group in the occipital cortex (channel 71, $\mathrm{p}=0.073$, Table 2).

\section{Discussion}

Inactive group had longer vMMN latencies in occipital channels when compared to active participants, yet frontal channels showed no group difference. Peak latencies illustrate the time taken by the nerve activity to travel and be processed in the involved cortical network and thus this indicates that the active participants had faster reaction and precognitive, automatic, processing for the randomly presented deviant visual stimuli. The vMMN integral values were measured from the rectified difference waves in the window of 100-300 ms. Area measured under the waveform represents the amount of cells activated and energy needed in the generation of waveform in question more accurately than peak amplitude. Thus the entire magnitude of the brain response in difference waveform is measured. There were no group differences in vMMN integrals, however, a trend for difference in left occipital recording site was observed. Faster latency and a tendency to increased integral together would therefore suggest somewhat more synchronized activity in the stellate cell groups, both simple and complex cells in cortical layers II-VI, which are likely in charge of precognitive visual processing in active individuals.

Previously it has been shown that vMMN can be found also in frontal areas (Kimura, 2012; Kremlacek et al., 2016). As we can see from the frontal difference waveforms (Fig. 2.), some deviant-related processing took place in the frontal lobe in both hemispheres. In our data, the 
latencies were longer (for example channel 20: $255 \pm 44 \mathrm{~ms}$ vs. channel 72: $224 \pm 34 \mathrm{~ms}$ ) in frontal areas than in the occipital lobe ( $\mathrm{p}=.003)$, which corresponds well to findings in earlier studies (Czigler, Balazs, \& Winkler, 2002; Winkler \& Czigler, 2012). The integrals in our frontal difference waveforms were smaller than those in the occipital region $(\mathrm{p}=.017)$. This suggests more of the synchronized neuronal activity taking place in the occipital lobe compared to the frontal lobe in vMMN.

VMMN has recently been explained with the predictive-coding theory (Kimura, 2012; Kimura \& Takeda, 2015; Stefanics et al., 2014). According to the theory, vMMN is a process resulting from a rule-violation. (Kimura \& Takeda, 2015). As the prediction-theory has been largely accepted as the background of vMMN, Kimura presents two possible theories to further complete the functional significance of vMMN (Kimura, 2012). In first theory, vMMN is considered as an attention triggering process for more extensive processes. This means that detecting events, that are incongruent to the prediction constructed by earlier events, is valuable for triggering attention that is required for further processing of the situation. The second theory describes $\mathrm{vMMN}$ as an updating of predictive models that is initiated by the detection of incongruent events. This would then allow more accurate predictions in the future.

It is generally recognized that vMMN is a result of detecting surprising events in the environment, without attention directed to them. Our survival has for the long run depended on this kind of ability, e.g. a falling tree, a predator nearby, or even a more contemporary problem, such as a danger in the road while driving a car. This kind of precognitive visual processing probably remains an important feature for us in everyday life. PA is widely known 
to be beneficial to our health, and an increasing evidence supports the idea that PA is beneficial to brain health and good cognitive performance. However, specific visual processing, such as vMMN detailed in the present study have not been connected with individual long term differences in PA.

\section{Conclusion}

Recently increasing evidence has demonstrated that PA has an effect on brain structure and function as well even in young age (Rottensteiner et al., 2015). Since PA is beneficial to cerebral vasculature, neurogenesis and synaptic plasticity (Nishijima et al., 2016; van Praag et al., 2014), it is likely that augmentation may occur in particular cortical precognitive processes, such as vMMN, as well. VMMN offers a way to study functionally complex but specific automatic cortical processes and there is evidence of altered $\mathrm{vMMN}$ in relation to cognitive decline and some neurological disorders as well (Kremlacek et al., 2016). However, there are no studies connecting PA and vMMN. Our study suggests that non-attend automatic visual processing may be enhanced by long term PA.

\section{References}

Andel, R., Crowe, M., Pedersen, N. L., Fratiglioni, L., Johansson, B., \& Gatz, M. (2008). Physical exercise at midlife and risk of dementia three decades later: A population-based study of swedish twins. The Journals of Gerontology.Series A, Biological Sciences and Medical Sciences, 63(1), 62-66. 
Astikainen, P., Lillstrang, E., \& Ruusuvirta, T. (2008). Visual mismatch negativity for changes in orientation--a sensory memory-dependent response. The European Journal of Neuroscience, 28(11), 2319-2324. doi:10.1111/j.1460-9568.2008.06510.x [doi]

Czigler, I., Balazs, L., \& Winkler, I. (2002). Memory-based detection of task-irrelevant visual changes. Psychophysiology, 39(6), 869-873. doi:10.1017/S0048577202020218 [doi]

Czigler, I., Weisz, J., \& Winkler, I. (2007). Backward masking and visual mismatch negativity: Electrophysiological evidence for memory-based detection of deviant stimuli. Psychophysiology, 44(4), 610-619. doi:PSYP530 [pii]

Donnelly, J. E., Hillman, C. H., Castelli, D., Etnier, J. L., Lee, S., Tomporowski, P., . . Szabo-Reed, A. N. (2016). Physical activity, fitness, cognitive function, and academic achievement in children: A systematic review. Medicine and Science in Sports and Exercise, 48(6), 1197-1222. doi:10.1249/MSS.0000000000000901 [doi]

Erickson, K. I., Prakash, R. S., Voss, M. W., Chaddock, L., Hu, L., Morris, K. S., . . Kramer, A. F. (2009). Aerobic fitness is associated with hippocampal volume in elderly humans. Hippocampus, 19(10), 1030-1039. doi:10.1002/hipo.20547; 10.1002/hipo.20547

Haapala, E. A., Vaisto, J., Lintu, N., Westgate, K., Ekelund, U., Poikkeus, A. M., . . Lakka, T. A. (2016). Physical activity and sedentary time in relation to academic achievement in children. Journal of Science and Medicine in Sport, doi:S1440-2440(16)30238-9 [pii]

Hillman, C. H., Erickson, K. I., \& Kramer, A. F. (2008). Be smart, exercise your heart: Exercise effects on brain and cognition. Nature Reviews.Neuroscience, 9(1), 58-65. doi: $10.1038 / \mathrm{nrn} 2298$

Kaprio, J., Pulkkinen, L., \& Rose, R. J. (2002). Genetic and environmental factors in healthrelated behaviors: Studies on finnish twins and twin families. Twin Research: The 
Official Journal of the International Society for Twin Studies, 5(5), 366-371.

doi:10.1375/136905202320906101 [doi]

Kimura, M. (2012). Visual mismatch negativity and unintentional temporal-context-based prediction in vision. International Journal of Psychophysiology : Official Journal of the International Organization of Psychophysiology, 83(2), 144-155.

doi:10.1016/j.ijpsycho.2011.11.010 [doi]

Kimura, M., \& Takeda, Y. (2015). Automatic prediction regarding the next state of a visual object: Electrophysiological indicators of prediction match and mismatch. Brain Research, 1626, 31-44. doi:10.1016/j.brainres.2015.01.013 [doi]

Kremlacek, J., Kreegipuu, K., Tales, A., Astikainen, P., Poldver, N., Naatanen, R., \& Stefanics, G. (2016). Visual mismatch negativity (vMMN): A review and meta-analysis of studies in psychiatric and neurological disorders. Cortex; a Journal Devoted to the Study of the Nervous System and Behavior, 80, 76-112. doi:10.1016/j.cortex.2016.03.017 [doi]

Kuldkepp, N., Kreegipuu, K., Raidvee, A., Naatanen, R., \& Allik, J. (2013). Unattended and attended visual change detection of motion as indexed by event-related potentials and its behavioral correlates. Frontiers in Human Neuroscience, 7, 476.

doi:10.3389/fnhum.2013.00476 [doi]

Naatanen, R., Gaillard, A. W., \& Mantysalo, S. (1978). Early selective-attention effect on evoked potential reinterpreted. Acta Psychologica, 42(4), 313-329. doi:00016918(78)90006-9 [pii]

Nishijima, T., Torres-Aleman, I., \& Soya, H. (2016). Exercise and cerebrovascular plasticity. Progress in Brain Research, 225, 243-268. doi:10.1016/bs.pbr.2016.03.010 [doi] 
Pazo-Alvarez, P., Cadaveira, F., \& Amenedo, E. (2003). MMN in the visual modality: A review. Biological Psychology, 63(3), 199-236. doi:S0301051103000498 [pii]

Rottensteiner, M., Leskinen, T., Niskanen, E., Aaltonen, S., Mutikainen, S., Wikgren, J., . . . Kujala, U. M. (2015). Physical activity, fitness, glucose homeostasis, and brain morphology in twins. Medicine and Science in Sports and Exercise, 47(3), 509-518. doi:10.1249/MSS.0000000000000437 [doi]

Smith, P. J., Blumenthal, J. A., Hoffman, B. M., Cooper, H., Strauman, T. A., Welsh-Bohmer, K., . . . Sherwood, A. (2010). Aerobic exercise and neurocognitive performance: A metaanalytic review of randomized controlled trials. Psychosomatic Medicine, 72(3), 239252. doi:10.1097/PSY.0b013e3181d14633 [doi]

Stefanics, G., Kremlacek, J., \& Czigler, I. (2014). Visual mismatch negativity: A predictive coding view. Frontiers in Human Neuroscience, 8, 666. doi:10.3389/fnhum.2014.00666 [doi]

Themanson, J. R., \& Hillman, C. H. (2006). Cardiorespiratory fitness and acute aerobic exercise effects on neuroelectric and behavioral measures of action monitoring. Neuroscience, 141(2), 757-767. doi:S0306-4522(06)00486-6 [pii]

van Praag, H., Fleshner, M., Schwartz, M. W., \& Mattson, M. P. (2014). Exercise, energy intake, glucose homeostasis, and the brain. The Journal of Neuroscience : The Official Journal of the Society for Neuroscience, 34(46), 15139-15149. doi:10.1523/JNEUROSCI.2814-14.2014 [doi]

Voelcker-Rehage, C., \& Niemann, C. (2013). Structural and functional brain changes related to different types of physical activity across the life span. Neuroscience and Biobehavioral Reviews, 37(9 Pt B), 2268-2295. doi:10.1016/j.neubiorev.2013.01.028; 10.1016/j.neubiorev.2013.01.028 
WHO. (2010). Global recommendations for physical activity for health. doi:NBK305057 [bookaccession]

Winkler, I., \& Czigler, I. (2012). Evidence from auditory and visual event-related potential (ERP) studies of deviance detection (MMN and vMMN) linking predictive coding theories and perceptual object representations. International Journal of Psychophysiology : Official Journal of the International Organization of Psychophysiology, 83(2), 132-143. doi:10.1016/j.ijpsycho.2011.10.001 [doi] 


\section{Figure legends}

Fig. 1.

VMMN grand average waveforms of deviant stimuli in 16 active participants (A) and in 16 inactive participants (B); all were young healthy males. All 128 channels are superimposed, referenced to $\mathrm{Cz}$ and topographic voltage distribution maps are shown at selected time points (100 ms, $122 \mathrm{~ms}, 143 \mathrm{~ms}, 190 \mathrm{~ms}, 211 \mathrm{~ms}$ and $245 \mathrm{~ms}) .0$ is the onset of stimulation.

Fig. 2.

VMMN waveforms shown in selected channels in active (A) and in inactive participants (B). In the middle is a schematic picture of the electrode net, where black indicates the illustrated channels. The channel numbers are in upper right hand corner of each box. Note, that the shaded area illustrates the analysed window. Blue waveforms=standard, red waveforms $=$ deviant, black waveforms $=$ difference. 
TABLE 1. Characteristics of participant groups (divided by their activity level, active group $\geq 2$ average daily MET-index $(n=16)$, inactive group $<2$ average daily MET-index $(n=16)$.

\begin{tabular}{lllll}
\hline & Active $( \pm$ SD $)$ & Inactive $( \pm$ SD $)$ & Mean difference & p-value \\
\hline Age & $34.0( \pm 1.5)$ & $34.4( \pm 1.4)$ & .37 & .402 \\
Aver dailyMET & $7.80( \pm 5.8)$ & $0.95( \pm 0.5)$ & 6.85 & $<.001^{* * *}$ \\
Height $(\mathrm{cm})$ & $180.3( \pm 5.3)$ & $174.6( \pm 9.2)$ & 5.78 & .138 \\
Weight $(\mathrm{kg})$ & $75.1( \pm 8.4)$ & $75.8( \pm 13.4)$ & 0.67 & .696 \\
BMI & $23.0( \pm 1.7)$ & $24.8( \pm 3.5)$ & 1.74 & .160 \\
Fat\% & $16.1( \pm 6.3)$ & $24.2( \pm 5.8)$ & 8.00 & $.002^{* *}$ \\
Waist circ. $(\mathrm{cm})$ & $82.5( \pm 6.4)$ & $88.8( \pm 9.9)$ & 6.25 & $.043^{*}$ \\
VO ${ }_{2}$ max & $48.5( \pm 9.0)$ & $37.2( \pm 5.4)$ & 11.29 & $<.001^{* * *}$ \\
$(\mathrm{ml} / \mathrm{kg} /$ min $)$ & & &
\end{tabular}

by Mann-Whitney U-test. 
TABLE 2. Mean peak latencies (ms) of two components in active $(n=16)$ and inactive $(n=16)$ groups in section A and vMMN integrals $(\mu \mathrm{V} / \mathrm{s})$ in $100-300 \mathrm{~ms}$ window in active $(n=16)$ and inactive $(n=16)$ groups in section B. Channel numbers from EGIS net indicate approximate International 10-20 System locations; 20=F3, 118=F4, 71=O1, 72=Pz and 76=O2.

A

\begin{tabular}{|c|c|c|c|c|c|c|c|c|}
\hline \multirow{3}{*}{$\begin{array}{l}\text { Chan- } \\
\text { nel }\end{array}$} & \multicolumn{4}{|c|}{ Early component } & \multicolumn{4}{|c|}{ Late component (vMMN) } \\
\hline & \multirow{2}{*}{\multicolumn{2}{|c|}{ Active \pm SD Inactive \pm SD }} & \multirow[t]{2}{*}{$\mathrm{Z}$} & \multirow[t]{2}{*}{$\mathrm{p}$-value } & \multirow[b]{2}{*}{ Active $\pm \mathrm{SD}$} & \multirow{2}{*}{$\begin{array}{l}\text { Inactive } \\
\pm \mathrm{SD}\end{array}$} & \multirow[t]{2}{*}{$\mathrm{Z}$} & \multirow[t]{2}{*}{$\mathrm{p}$-value } \\
\hline & & & & & & & & \\
\hline 20 & $134 \pm 28$ & $144 \pm 31$ & -0.99 & .341 & $255 \pm 44.3$ & $255 \pm 46.7$ & 0.27 & .809 \\
\hline 118 & $151 \pm 27$ & $152 \pm 28$ & 0.00 & 1.00 & $248 \pm 47.0$ & $252 \pm 45.0$ & -0.06 & .956 \\
\hline 71 & $140 \pm 20$ & $148 \pm 23$ & -1.01 & .323 & $207 \pm 23.4$ & $248 \pm 34.9$ & -3.24 & $.001 * *$ \\
\hline 72 & $144 \pm 17$ & $143 \pm 24$ & 0.06 & .956 & $211 \pm 27.0$ & $239 \pm 35.7$ & -2.26 & $.023 *$ \\
\hline 76 & $147 \pm 22$ & $145 \pm 20$ & 0.35 & .752 & $213 \pm 22.9$ & $243 \pm 37.7$ & -2.23 & $.026 *$ \\
\hline \multicolumn{9}{|l|}{ B } \\
\hline 20 & $0.26 \pm 0.14$ & $0.19 \pm 0.09$ & 1.39 & .171 & & & & \\
\hline 118 & $0.20 \pm 0.12$ & $0.18 \pm 0.08$ & 0.15 & .897 & & & & \\
\hline 71 & $0.35 \pm 0.19$ & $0.24 \pm 0.09$ & 1.81 & .073 & & & & \\
\hline 72 & $0.28 \pm 0.19$ & $0.20 \pm 0.05$ & 1.17 & .254 & & & & \\
\hline 76 & $0.36 \pm 0.22$ & $0.27 \pm 0.13$ & 1.17 & .254 & & & & \\
\hline
\end{tabular}

by Mann-Whitney U-test. 